\title{
A Decentralized Deadline-Driven Electric Vehicle Charging Recommendation
}

\author{
Yue Cao ${ }^{\circledR}$, Member, IEEE, Omprakash Kaiwartya ${ }^{\circledR}$, Member, IEEE, Yuan Zhuang ${ }^{\circledR}$, Member, IEEE, \\ Naveed Ahmad, Yan Sun ${ }^{\circledR}$, and Jaime Lloret ${ }^{(0)}$, Senior Member, IEEE
}

\begin{abstract}
The electric vehicle (EV) industry has been rapidly developing internationally due to a confluence of factors, such as government support, industry shifts, and private consumer demand. Envisioning for the future connected vehicles, the popularity of EVs will have to handle a massive information exchange for charging demand. This inevitably brings much concern on network traffic overhead, information processing, security, etc. Data analytics could enable the move from Internet of EVs to optimized EV charging in smart transportation. In this paper, a mobile edge computing (MEC) supporting architecture along with an intelligent $\mathrm{EV}$ charging recommendation strategy is designed. The global controller behaves as a centralized cloud server to facilitate analytics from charging stations (CSs) (service providers) and charging reservation of on-the-move EVs (mobile clients) to predict the charging availability of CSs. Besides, road side units behave as MEC servers to help with the dissemination of the CSs' charging availability to EVs, and collecting their charging reservations, as well as operating decentralized computing on reservations mining and aggregation. Evaluation results show the features of the MEC-based charging recommendation system in terms of communication efficiency (low cost for information dissemination and collection) and improvement of charging performance (reduced charging waiting time and increased fully charged EVs).
\end{abstract}

Index Terms-Charging recommendation, electric vehicle (EV), mobile edge computing (MEC), Vehicle-to-Infrastructure.

\section{INTRODUCTION}

$\mathbf{T}$ HE introduction of electric vehicles (EVs) [1] will have a significant impact on the sustainable economic development of urban cities. However, even if there have been charging service providers available, the utilization of charging infrastructures is still in need of significant enhancement. Such a situation certainly requires the popularity of EVs toward the

Manuscript received February 6, 2018; revised April 21, 2018; accepted June 23, 2018. (Corresponding author: Yuan Zhuang.)

Y. Cao is with the Department of Computer and Information Sciences, Northumbria University, Newcastle upon Tyne NE1 8ST, U.K. (e-mail: yue. caoa@northumbria.ac.uk).

O. Kaiwartya is with the School of Science and Technology, Nottingham Trent University, Nottingham NG1 4FQ, U.K. (e-mail: omprakash. kaiwartya@ntu.ac.uk).

Y. Zhuang is with the Bluvision, Inc., Part of HID Global, Fort Lauderdale, FL 33334 USA (e-mail: zhy.0908@gmail.com).

N. Ahmad is with the Department of Computer Science, University of Peshawar, Peshawar 25000, Pakistan (e-mail: n.ahmad@upesh.edu.pk).

Y. Sun is with the School of Electronic Engineering and Computer Science, Queen Mary University of London, London E1 4NS, U.K. (e-mail: yan.sun@qmul.ac.uk).

J. Lloret is with the Universitat Politecnica de Valencia, Valencia 46022, Spain (e-mail: jlloret@dcom.upv.es).

Digital Object Identifier sustainable, green, and economic market. Enabling the sustainability requires a joint contribution from each domain, e.g., how to schedule charging services for EVs being parked within the grid capacity, how to optimally recommend $\mathrm{EV}$ drivers toward the charging station (CS) with the least waiting time, and how to guarantee accurate information involved in decision making.

Unlike many previous works [2] that investigate "charging scheduling" (referred to when/whether to charge) for EVs already been parked at CSs, a few recent works focus on "charging recommendation" (refer to where/which CS to charge) [3] for on-the-move EVs. The latter case has been the most important feature of improving the charging Quality of Experience (QoE), as applied by operators. Thus, it is important to optimally recommend EV drivers regarding where to charge, concerning the service waiting time.

Literature works [4]-[8] have addressed the charging recommendation to improve the charging QoE (e.g., to reduce the service waiting time for charging). Usually, the local condition of CSs (e.g., number of EVs being parked and their remaining charging time) [7] is considered to make a charging recommendation decision. Further advanced solutions utilize the EV's charging reservation [1], [9]-[11] to align with the local condition of CSs. By doing so, it can be predicted at what time and which CS will be congested, so as not be recommended for charging. Here, the charging reservation includes the arrival time (when an EV will arrive at the recommended CS) and the expected charging time at the selected CS (how long its charging time will be).

Practically, EV drivers would also have their parking deadline [11] at CSs (e.g., drivers might be impatient to wait for a long time, or have another daily agent after a certain period of charging). Particularly, in the case of charging during peak time, already deployed charging slots at CSs may not be sufficient to handle such an urgent charging demand (due to limited parking duration). Inevitably, an inappropriate charging recommendation would degrade the charging QoE, as some EVs will have to leave after the deadline even though they have not been charged. Consequently, charging will involve additional effort and energy consumption; such an inconvenience would, however, discourage the willingness to switch from traditional vehicles to EVs.

The centralized cloud (CC) based system [12] is widely applied in the literature for charging recommendation. Such a system generally relies on ubiquitous cellular network and real-time information for optimization. For example, previous work [11] adopted a cloud-based global controller (GC) connecting to all 
CSs. Whenever an EV requires charging, it will send a request to the GC through the cellular network seeking the best CS recommendation, and further reports its charging reservation. By facilitating the anticipated EV charging recommendation, the charging availability of the CS can be predicted, so that the cloud will not recommend a CS with low availability.

However, by seamlessly collecting information from EVs and CSs, it is very time consuming for the GC to achieve optimization. The complexity and computation load of the cloud server increases exponentially (depends on those who currently request charging and those who have made charging reservations) with the number of EVs. Moreover, the cellular network is costly and sometime overcongested due to massive accesses, which degrades the quality of communication. The rapid growth of mobile applications has placed severe demands on the cloud infrastructure, which has led to moving computing and data services toward the edge of the cloud, resulting in a novel mobile edge computing (MEC) [13] (also known as fog computing) architecture being developed by the European Telecommunications Standards Institute (ETSI) and creating a new Industry Specification Group in 2014 for this purpose. MEC could reduce data transfer times, remove potential performance bottlenecks, and increase data security and enhance privacy while enabling advanced applications.

As such, in the case of EV charging, a decentralized charging recommendation with the assistance of MEC servers positioned close to EVs is desirable. Apart from the cellular network, a cheaper solution nowadays is the deployment of fixed road side units (RSUs) [14] based on license-free spectrum such as Wi-Fi, but only with limited network coverage. Future intelligent transportation systems (ITS) [15] will necessitate infrastructure-assisted communication for EV charging perspective in addition to road safety perspective. In [10], a decentralized MEC-based information communication technology (ICT) framework has been proposed where it facilitates the RSUs (with MEC servers) to perform information caching, aggregation, and lightweight processing (e.g., access control and information mining); system level communication cost within the charging recommendation system can be reduced. Besides, by cooperating with the cloud server GC, deployed RSUs also help to disseminate and collect information between CSs and EVs ubiquitously.

Understandably, the integration of ICT, transport, and energy is important for the attainability of EV charging [16], [17]. This paper mainly tackles a joint study of former transport planning and ICT, whereas the integration of energy substainability (e.g., smart charging, scheduling of renewable energy) is out of the scope. Beyond the ICT effort investigated in [10], we further take the impact of parking deadline and the decentralized ICT framework into account for the EV charging recommendation decision. More specifically, the EV's parking deadline will influence the estimation of CSs' charging queueing and prediction of their charging availability (in line with EVs' charging reservations collected through the positioned MEC architecture). In particular, the proposed solution on predicting the charging availability is decoupled and associated with a number of time intervals (within a dynamically updated time window). Such a feature benefits the accuracy of the charging recommendation, bounded by a prediction time window and EV mobility.

\section{RELATED WORK}

\section{A. Cloud/Mobile Edge Computing in Smart Transportation}

Smart transportation can fundamentally change urban lives at many levels. Data from service providers and users bridged via a ubiquitous, dynamic, scalable, and sustainable ecosystem would offer a wide range of benefits and opportunities. Most of the existing techniques require a high processing time using conventional methods of data processing [18]. Therefore, the techniques are desirable to efficiently process the data generated from stakeholders, ideally from a distributed manner through ubiquitously disseminated and collected information.

The major difference between cloud computing [12] and MEC [13] is in the location awareness to support application services. This is because the cloud server locates in a centralized place and behaves as a centralized manager to perform computation tasks. Note that MEC servers at different locations can be owned and managed by separate operators and owners. With the collaboration among different operators, they can form a collaborative and decentralized computing system in a wide region.

\section{B. EV Charging Recommendation}

As reviewed by the most recent survey [3], fruitful literature works have addressed "charging scheduling" [2], via regulating the $\mathrm{EV}$ charging, such as minimizing peak load/cost, flattening aggregated demands, or reducing frequency fluctuations.

In recent years, the "charging recommendation" problem has started to gain interest from industries thanks to the popularity of EVs. The generic solutions [4], [7] make decisions based on the queueing information at CSs, and the one with the minimum queueing time is recommended. This feature has been evaluated in [5] against the charging recommendation just taking the closest distance to the CS; the former is deemed as an effective guidance in an urban city with limited charging infrastructures. The charging recommendation solution in [8] adopts a pricing strategy to minimize congestion and maximize profit, by adapting the price depending on the number of EVs charging.

Beyond that, the integration of the ICT and energy network is of importance for the sustainability of EV charging, where a set of works have addressed the constraint of energy network and study its impact. From the ICT aspect, additional communication signaling is built to support the advanced charging recommendation and brings the anticipated EVs mobility information (charging reservations). The work in [9] concerns a highway scenario where the EV will pass through all CSs. The expected charging waiting time is calculated for the EV passing through the entire highway, by jointly considering the charging waiting time at a CS where the EV needs charging for the first time and the time spent at subsequent CSs, before exiting the highway. Other works [1], [10], [11] focus on urban city scenarios, where the EV travels toward a single geographically distributed CS for 
charging. The expected waiting time for charging is associated with that CS, rather than a subsequent charging in the case of the highway.

\section{PRovisioning OF MEC-BASEd Charging RECOMMENDATION SYSTEM}

In this section, we mainly introduce entities and system signaling of the proposed MEC-based system, together with an analysis on its advantage.

\section{A. Charging System Cycle}

Driving: This happens when the EV is traveling on the road (following a route in the city).

Charging Recommendation: If an EV's remaining electricity is below the state of charge (SOC) threshold value, the charging recommendation is required to guide it on where to charge.

Charging Scheduling: This happens when EVs have reached a CS. The CS implements a certain policy to schedule which EV is to be charged. Here, the first come first serve (FCFS) is widely applied in the problem of charging recommendation, where the EV with the earliest arrival time is scheduled as the highest priority.

Battery Charging: This phase reflects a continuous procedure to charge EVs, until they are fully charged. After that, those fully charged EVs will resume to the Driving Phase.

Typically, the system is a status transfer within four phases, while the Charging Scheduling has been extensively covered by the literature. The focus of this paper is on Charging Recommendation with interdisciplinary efforts from ICT.

\section{B. Network Entities}

1) Stakeholders: The EV below the SOC threshold (a value under which the EV should seek charging) needs to find a $\mathrm{CS}$ for charging. As long as the EV has been recommended to charge at a CS, the EV further reports its charging reservation associated with that $\mathrm{CS}$.

The CS is equipped with a number of plug-in charging slots to charge multiple EVs in parallel. Particularly, its local queuing information is monitored by the cloud server $\mathrm{GC}$ to compute the charging availability. This refers to the earliest time when a charging slot of the CS is unoccupied.

2) Cloud server: It is a logical server that is built and delivered through a cloud computing platform over CSs and EVs. Here, the GC manages the CSs' charging availability, based on the monitored CSs' local queueing information, and EVs' charging reservations (collected by MEC servers).

3) MEC server: The MEC servers collected at RSUs provide a set of middle-ware services associated with applications, wherein it implements two key operations as follows.

a) Disseminate CSs' charging availability (computed by the GC) to EVs.

b) Enable information mining and aggregation (complementarily with authentication) for opportunistically collected EVs' charging reservations.

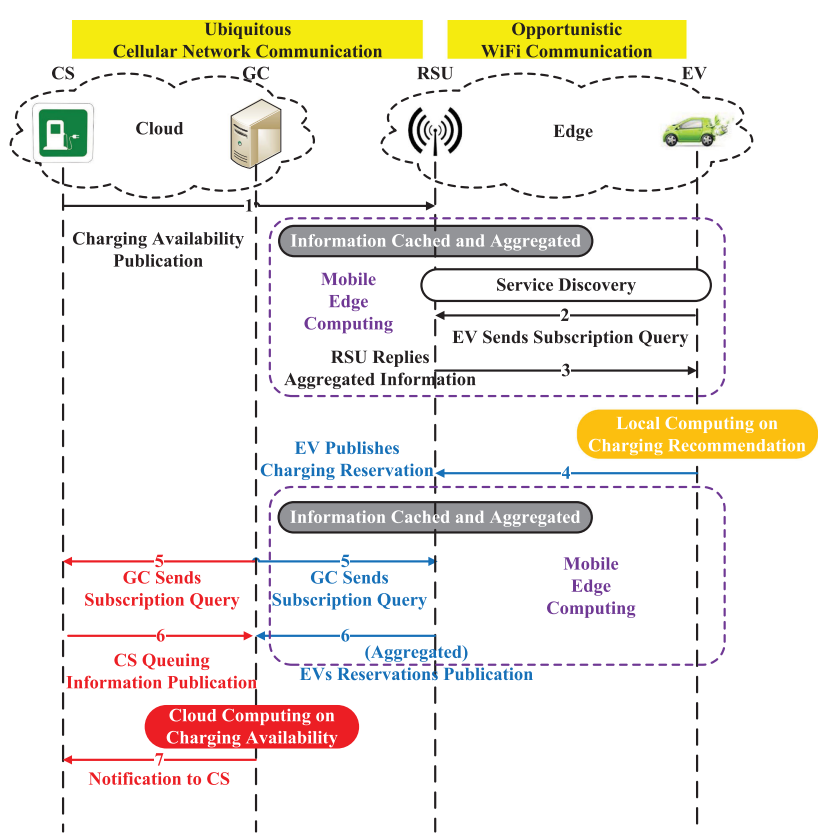

Fig. 1. Signaling process for the MEC-based system.

TABLE I

COMMUNICATION TECHNOLOGIES IN MEC- AND Cloud-BASED Systems

\begin{tabular}{|l|l|l|l|l|}
\hline & $\begin{array}{l}\text { GC } \leftrightarrow \text { MEC } \\
\text { Server }\end{array}$ & GC $\leftrightarrow$ CS & $\begin{array}{l}\text { MEC } \\
\text { Server } \leftrightarrow \mathbf{E V}\end{array}$ & GC $\leftrightarrow \mathbf{E V}$ \\
\hline $\begin{array}{l}\text { MEC- } \\
\text { based } \\
\text { System }\end{array}$ & $\begin{array}{l}\text { Internet, Cellu- } \\
\text { lar network }\end{array}$ & $\begin{array}{l}\text { Internet, Cellu- } \\
\text { lar network }\end{array}$ & $\begin{array}{l}\text { WiFi commu- } \\
\text { nication }\end{array}$ & N/A \\
\hline $\begin{array}{l}\text { Cloud- } \\
\text { based } \\
\text { System }\end{array}$ & N/A & N/A & N/A & $\begin{array}{l}\text { Cellular } \\
\text { network }\end{array}$ \\
\hline
\end{tabular}

\section{Communication Technologies}

As shown in Fig. 1, the communication technology applied between GC and CSs can be simply based on reliable Internet or cellular network, mainly because they are fixed network entities. However, there is a necessity to scalably and ubiquitously disseminate CSs' charging availability (computed by the GC) to EVs, and collect EVs' charging reservations. Although 3G/LTE can be applied thanks to ubiquitous coverage, EVs' charging requests are just on-demand, whereas CSs charging availability is fluctuated within certain periods (e.g., minutes level). Besides, EVs' charging reservations are generated only when they have been given the charging recommendation. This motivates the application of short-range and on-demand communication with EVs. Motivated by the above-mentioned discussion, the opportunistic communication paradigm, e.g., delay/disruption tolerant networking [19], between EVs and MEC servers is desirable, which alleviates the burden of solely relying on the cellular network. Table I summarizes communication technologies in MEC- and cloud-based systems.

Furthermore, rather than using the point-to-point-based communication, the topic-based communication (e.g., publish/subscribe pattern [20]) mainly offers communications decoupled in space that subscribers do not need to know the 
TABLE II

TOPICS DEFINED IN THE MEC-BASED SYSTEM

\begin{tabular}{|l|l|l|l|}
\hline Topic & Publisher & Subscriber & Payload \\
\hline CA_Update & CS & RSUs & <CS ID, CS's charging availability, dissemination time slot $>$ \\
\hline Aggregated_CA_Update & RSUs & EVs & $\begin{array}{l}<\text { Aggregated CS IDs and CSs' charging availability, dissem- } \\
\text { ination time slot }>\end{array}$ \\
\hline Charging_Reservations_Update & $\begin{array}{l}\text { EVs been recom- } \\
\text { mended charging }\end{array}$ & RSUs & $\begin{array}{l}<\text { EV ID, parking duration, arrival time, expected charging } \\
\text { time }>\end{array}$ \\
\hline Aggregated_Charging_Reservations_Update & RSUs & GC & $<$ Aggregated EVs' reservations cached by RSUs $>$ \\
\hline Local_Queuing_Update & CSs & GC & $\begin{array}{l}<\text { CS's local queuing information, including number of EVs } \\
\text { being parked at CS and their charging time }>\end{array}$ \\
\hline CA_Prediction & GC & CSs & $<$ Predicted charging availability of each CS $>$ \\
\hline
\end{tabular}

location and address of publishers and vice versa. It is potentially in time as the system is able to store events for clients who are temporally disconnected.

The solutions to achieve trusted message exchange for the case of EV charging is to encrypt the sensitive information and hide the real identity. One development aspect of the encryption involves the light-weight and highly secured encryption algorithm, while another one is to design an efficient and scalable key management scheme. As for the privacy, a pseudonym is proposed to hide the identities. This includes the pseudonym changing algorithms and pseudonym reuse schemes, both are required to be implemented in efficient and scalable manners. The future challenges based on the MEC system are considered based on the nature of a large number of connected EVs, high mobility, wide coverage area, and heterogeneous communication systems.

\section{Proposed MEC-Based System}

It is assumed that the locations of all CSs are already known by EVs, e.g., through the vehicle on-board unit. Here, EVs access CSs' charging availability from MEC servers, make a local charging recommendation, and further report charging reservations (through MEC servers to the GC). The GC analyzes the EVs' charging reservations together with CSs' local queuing information to predict the CSs' charging availability. Fig. 1 illustrates a typical procedure.

Step 1: The GC periodically (with time interval $\Delta$ ) disseminates its computed CSs' charging availability to all legitimate MEC servers (positioned at RSUs), via "CA_Update" topic defined in Table II. RSUs further aggregate the information from all CSs and get cached. Note that the information disseminated at the previous $\Delta$, which to be further cached at MEC servers, will be replaced with the one that associates with the current $\Delta$. This guarantees the information accuracy involved for charging recommendation. The RSU receiving the dissemination from all CSs will aggregate and cache their information.

Steps 2 and 3: Upon encountering an RSU, the EV would subscribe to the cached information from the RSU through the P/S system. In particular, the EV only subscribes to the information that is recently published using the "Aggregated_CA_Update" topic. This reduces the redundant access signaling, particularly when an EV frequently encounters several RSUs in a short time (still within the current dissemination interval $\Delta$ ). For example, if an EV has already obtained information from $\mathrm{RSU}_{1}$ within

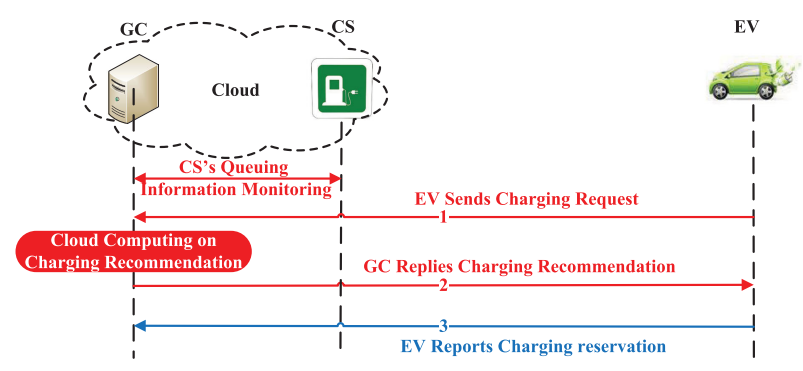

Fig. 2. Signaling process for the CC-based system.

interval $\Delta$, its subscription will be denied by $\mathrm{RSU}_{2}$ within the same interval.

Step 4: The EV makes a charging recommendation in the case of low energy status and publishes its charging reservation to any encountered MEC server along the road. Here, the "Charging_Reservations_Update" topic is applied, with the EV as publisher and RSUs (MEC servers) as subscribers. Each RSU mines the valid EV's charging reservation and aggregates them. The valid charging reservation refers to that of which EV's arrival is supposed to be later than $(\Delta+\mathcal{P})$, where $\mathcal{P}$ is the time slot of the previous dissemination. This is because an EV's reservation will be deleted by its selected CS when it is parked therein. Then, any arrival occurring before the next dissemination will be removed from RSUs; this potentially reduces the size of data to be uploaded to the GC.

Steps 5 and 6: At the GC side, it sets two separate topics to collect information from CSs and RSUs.

1) The local condition of CSs includes the number of EVs being parked and their required battery charging time. This is accessible by sending a subscription via the " $\mathrm{LO}$ cal_Queuing_Update" topic.

2) The GC also accesses the aggregated EVs' charging reservations from all RSUs, using the "Aggregated_Charging_Reservations_Update" topic.

Step 7: The GC then predicts the charging availability of CSs and pushes them for dissemination at the following time slot, using the "CA_Prediction" topic.

\section{E. Other Alternative Systems}

1) CC-Based System: It is implemented in a centralized manner in the cloud system, as shown in Fig. 2. 


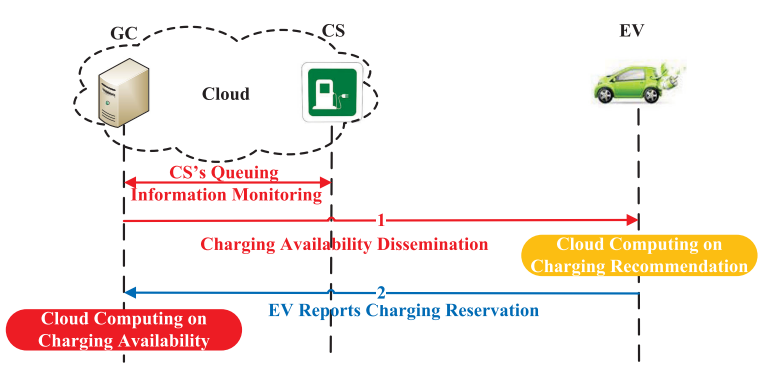

Fig. 3. Signaling process for the DC-based system.

Step 1: The EV, which needs charging, sends its charging recommendation request to the $\mathrm{GC}$ through the cellular network.

Step 2: Upon receiving the request from an EV, the GC makes a charging recommendation based on the intelligence proposed in Section IV, and further replies back to the pending EV.

Step 3: The EV that accepts the decision, then starts a journey toward the recommended CS. Meanwhile, it reports its charging reservation to the $\mathrm{GC}$, so that the GC can estimate the occultation of the reserved CS in the near future.

2) Decentralized Cloud (DC) Based System: This is the distributed version of the CC-based system (based on cellular network), as shown in Fig. 3.

Step 1: Each CS periodically (with interval $\Delta$ ) broadcasts its charging availability to all EVs, also through the cellular network communication. This mechanism also equals the case that each EV subscribes to CS's charging availability from the $\mathrm{GC}$, through topic-based P/S communication, where there is no RSU involved to help decentralize the global computation.

Step 2: The EV individually makes charging recommendation and reports its charging reservation to the GC through the same communication channel. Upon directly receiving the EV's charging reservations and continuously monitoring the CSs' local queuing information, the GC predicts the charging availability of CSs and notifies them for dissemination the next time around.

\section{F. Discussion}

Denoting $N_{\mathrm{ev}}, N_{\mathrm{mec}}$, and $N_{\mathrm{cs}}$ as the number of EVs, MEC servers, and CSs, respectively, the communication costs of the MEC- and cloud-based systems are analyzed as follows.

MEC-Based System: As shown in Fig. 1, the delay is mainly from the time for the EV to encounter an RSU, as the communication between RSUs and GC is through cellular network or Internet. Therefore, the dissemination cost is scaled by $O\left(\Theta \times N_{\mathrm{ev}}\right)$; recall that $\Theta$ is the possibility that an EV encounters at least one of $N_{\text {mec }}$ RSUs [10]

$$
\Theta \leq 1-\prod_{i=1}^{N_{\mathrm{mec}}}\left\{1-\left[\frac{(i-1) X+F+R}{S \cdot \Delta}\right]\right\}
$$

where $X$ is the distance between adjacent RSUs, and $S$ is the EV speed, $R$ is the V2I communication range, while $F$ is a constant that shows the distance from the EV to the first RSU. Note that $R$ depends on the transmission power and other practical configurations at the EV side, as it is the initiator to establish communication with the RSU for information subscription.

Next, concerning aggregated EVs' reservations uploading to the GC before $(\Delta+\mathcal{P})$, the reservation cost is scaled by $O\left(\frac{N_{\text {mec }}}{\Delta}\right)$, as the communication is established from $N_{\text {mec }}$ RSUs within interval $\Delta$. As such, excluding the deployment of RSUs, in nature, a larger $N_{\mathrm{ev}}$ drives the sustainable communication efficiency for the long-term popularity of EVs.

$C C$-Based System: The GC experiences a cost of $O\left(N_{\mathrm{ev}}\right)$ for handling the charging requests/reservations from $N_{\mathrm{ev}} \mathrm{EVs}$.

$D C$-Based System: The GC experiences a cost of $O\left(\frac{N_{\mathrm{ev}}}{\Delta}\right)$ for periodically disseminating the CS's charging availability, and $O\left(N_{\mathrm{ev}}\right)$ for handling EVs' charging reservations.

The CC-based system suffers from privacy concerns, in which the driving behavior (e.g., location) has to be included when communicating with the GC (see Step 1 in Fig. 2). Besides, the DC-based system does not involve MEC servers; it, however, relies on the broadcast communication feature under the environment of a ubiquitous cellular network. This is much expensive than the MEC-based system, as the latter just requires a short-range wireless communication network between MECs servers and a large number of EVs. In reality, the number of RSUs is less than that of EVs, given by $\left(N_{\text {mec }} \ll N_{\text {ev }}\right)$. However, the number of charging services is higher than the actual number of EVs $N_{\mathrm{ev}}$. This is because each EV needs to charge more than once. This claims the communication efficiency of MEC-based system over CC-based system.

\section{DESIGN OF CHARGING RECOMMENDATION}

Previous works [9], [11] have proposed the formulation on how to minimize the charging waiting time for all EVs in the network. Generally, an even distribution of EVs among CSs contributes to the minimized charging waiting for EVs. In the following part, the proposed charging recommendation solution is presented through the decentralized manner that is applicable to the MEC-based ICT framework. Note that the proposed solution focuses on how to distribute EVs among all CSs in a decentralized manner (through the ICT framework), while any user-driven solution by taking into consideration the trip destination and pricing will be of interest in further studies.

In Fig. 4, the CS's charging availability is predicted without/with EVs' charging reservations (shown in Table IV), as detailed in Algorithm 3 (requires the estimation of CS's local queuing from Algorithm 2) and Algorithm 4, respectively. Then, Algorithm 1 will produce the CS's charging availability associated with each time slot, where these time slots are decoupled from an estimation time window $\mathcal{W}$. With this knowledge disseminated from CSs, the EV locally makes a charging recommendation, via the output of Algorithm 5.

As the estimation of charging availability per CS depends on whether there have been EVs remotely reserved for charging, such complexity is $O\left(N_{\mathrm{ev}}^{2}\right)$ since both the EVs locally parked and those remotely reserved are considered in Algorithm 4. In Algorithm 3, the complexity is $O\left(N_{\mathrm{ev}}\right)$ as there is no EV reserved for charging. All notations are defined in Table III. 


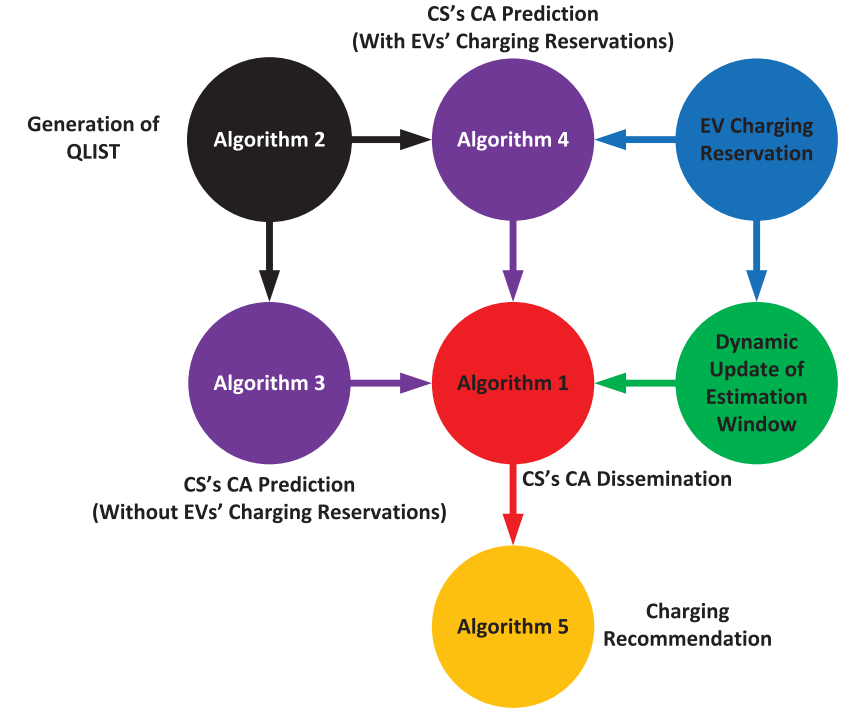

Fig. 4. Process flow of charging recommendation.

TABLE III

LIST OF NOTATIONS

\begin{tabular}{|c|l|}
\hline$\Delta$ & Charging availability dissemination interval \\
\hline$S_{e v}$ & Moving speed of EV \\
\hline$\alpha$ & Electric energy consumed per meter \\
\hline$T_{c u r}$ & Current time in the network \\
\hline$\delta$ & Number of charging slots at CS \\
\hline$N_{W}$ & Number of EVs waiting for charging at CS \\
\hline$N_{C}$ & Number of EVs under charging at CS \\
\hline$E_{e v}^{m a x}$ & Full volume of EV battery \\
\hline$E_{e v}^{c u r}$ & Current volume of EV battery \\
\hline$\beta$ & Charging power at CS \\
\hline$T_{e v}^{f i n}$ & Charging finish time of EV \\
\hline$T_{e v}^{a r r}$ & EV's arrival time at CS \\
\hline$T_{e v}^{t r a}$ & EV's travelling time to reach CS \\
\hline$T_{e v}^{c h a}$ & Expected charging time upon arrival of EV \\
\hline$N_{R}$ & Number of EVs reserved for charging at CS \\
\hline $\mathcal{W}$ & Prediction time window \\
\hline $\mathcal{H}$ & Number of entries within $\mathcal{W}$ \\
\hline $\mathcal{K}$ & A time slot of $\mathcal{H}$ \\
\hline CA $\mathcal{K}$ & The given charging availability at $\mathcal{K}$ \\
\hline$D_{e v}$ & Time duration that EV will park at CS \\
\hline RLIST & Set including a number EVs made charging reservation at CS \\
\hline QLIST & Set including available time per charging slot at CS \\
\hline & \\
\hline
\end{tabular}

TABLE IV

CHARGING RESERVATION FORMAT

\begin{tabular}{|l|l|l|l|l|}
\hline EV ID & Selected CS & $\begin{array}{l}\text { Parking Dead- } \\
\text { line }\end{array}$ & Arrival Time & $\begin{array}{l}\text { Expected } \\
\text { Charging } \\
\text { Time }\end{array}$ \\
\hline $\mathrm{EV}_{1}$ & $\mathrm{CS}_{1}$ & $1200 \mathrm{~s}$ & $17260 \mathrm{~s}$ & $892 \mathrm{~s}$ \\
\hline
\end{tabular}

\section{A. EV's Charging Reservation}

The EV's charging reservation is generated from the EV that had made the charging recommendation and relayed through the MEC servers to the GC. As an example in Table IV, such information normally includes the ID of the recommended CS, the EV's parking deadline, arrival time at that CS, and the EV's expected charging time there, specifically as shown.

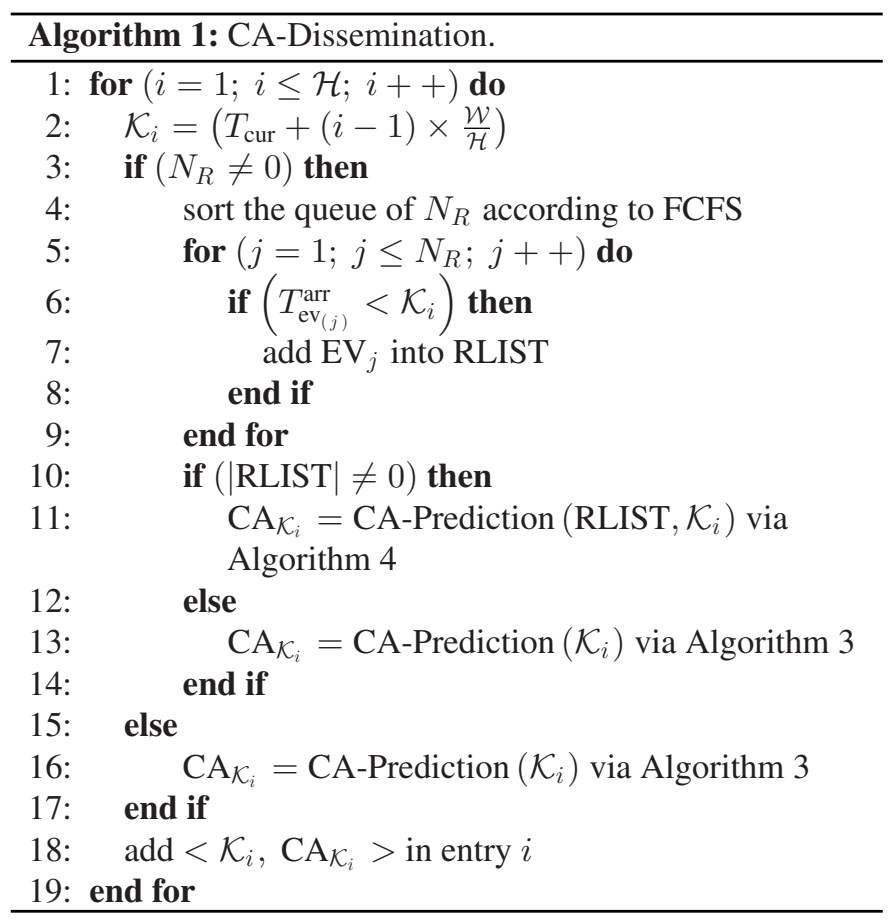

Arrival Time: The arrival time $T_{\mathrm{ev}}^{\mathrm{arr}}$ reflects the time when an $\mathrm{EV}$ reaches the recommended CS, where the value counts for the traveling time $T_{\mathrm{ev}}^{\text {tra }}$ from the current location of EV to the recommended CS

$$
T_{\mathrm{ev}}^{\mathrm{arr}}=T_{\mathrm{cur}}+T_{\mathrm{ev}}^{\mathrm{tra}}
$$

Expected Charging Time: The expected charging time $T_{\mathrm{ev}}^{\text {cha }}$ at the selected CS is given by

$$
T_{\mathrm{ev}}^{\mathrm{cha}}=\frac{E_{\mathrm{ev}}^{\mathrm{max}}-E_{\mathrm{ev}}^{\mathrm{cur}}+S_{\mathrm{ev}} \times T_{\mathrm{ev}}^{\mathrm{tra}} \times \alpha}{\beta} .
$$

Here, $\left(S_{\mathrm{ev}} \times T_{\mathrm{ev}}^{\mathrm{tra}} \times \alpha\right)$ is the energy consumed for the movement traveling to the selected CS, based on a constant $\alpha$ (depending on a certain type EV) measuring the energy consumption per meter.

Parking Deadline: $D_{\mathrm{ev}}$ is defined as a limitation on how long an EV will stay to wait for charging at the recommended CS.

\section{B. Charging Availability Dissemination}

Upon receiving EVs' charging reservations, each GC computes the charging availability for all connected CSs, associated with a number of time slots $\mathcal{K}$ that is beyond the interval $\Delta$. Here, given that there are predefined $\mathcal{H}$ time slots associated within $\mathcal{W}$, the gap between adjacent $\mathcal{K}$ time slots is calculated by $\frac{\mathcal{W}}{\mathcal{H}}$.

Algorithm 1 is implemented by the GC and disseminates information formatted in Table $\mathrm{V}$. The time slot at the $i$ th entry is calculated by $\mathcal{K}_{i}=\left(T_{\text {cur }}+(i-1) \times \frac{\mathcal{W}}{\mathcal{H}}\right)$, where $T_{\text {cur }}$ is the current time in the network. Understandably, $\mathcal{K}_{i}$ indicates a time slot beyond the current network time $T_{\text {cur }}$. An entire process of CS's information dissemination is presented as follows. 
TABLE V

FORMAT OF CS's CHARGING AVAILABILITY DISSEMINATION

\begin{tabular}{|c|c|c|}
\hline \multicolumn{3}{|c|}{$-\mathrm{CS}_{3}$} \\
\hline \multicolumn{3}{|c|}{ 10800s } \\
\hline \hline \multicolumn{3}{|c|}{-Dissemination Time Slot- } \\
\hline \multicolumn{3}{|c|}{} \\
\hline \multicolumn{3}{|c|}{-Charging Availability Predicted Within $\mathcal{W}-$} \\
\hline Entry & Decoupled Time Slot & Charging Availability \\
\hline 1 & $10860 \mathrm{~s}$ & $10892 \mathrm{~s}$ \\
\hline 2 & $10920 \mathrm{~s}$ & $10920 \mathrm{~s}$ \\
\hline 3 & $10980 \mathrm{~s}$ & $10980 \mathrm{~s}$ \\
\hline
\end{tabular}

1) The $\mathrm{EV}_{j}$ (in the queue of $N_{R}$ ), which has reported charging reservation to the recommended CS (while its arrival time $T_{\mathrm{ev}_{(j)}}^{\text {arr }}$ is earlier than $\mathcal{K}_{i}$ ), will be recorded into a list, namely RLIST. Here, we consider that there will be other EVs (in the queue of $N_{R}$ ) that reserve and reach at the same CS before the time slot $\mathcal{K}_{i}$ as the condition $\left(T_{\mathrm{ev}_{(j)}}^{\mathrm{arr}}<\mathcal{K}_{i}\right)$ at line 6. In this context, the charging availability estimated at $\mathcal{K}_{i}$, as denoted by $\mathrm{CA}_{\mathcal{K}_{i}}$, is calculated via Algorithm 4.

Note that, at line 11, the prediction of the CS's charging availability via Algorithm 4 requires an input of charging reservations of those $\mathrm{EV}_{j}$ with an earlier arrival time than $\mathcal{K}_{i}$. This is given by the condition at line 10 in Algorithm 1. Otherwise, Algorithm 3 is applied by only examining the local conditions of CSs (e.g., number of EVs being parked and remaining charging time).

2) Alternatively, Algorithm 3 is also applied if there are no EVs' charging reservations, as presented between lines 15 and 16.

Then, a pair of $\left\langle\mathcal{K}_{i}, \mathrm{CA}_{\mathcal{K}_{i}}\right\rangle$ stating the "〈time slot, charging availability at time slot $\rangle$ " will be prepared for dissemination. The information is then disseminated as shown in Step 1 in Fig. 1.

\section{Dynamic Update of $\mathcal{W}$}

Note that $\mathcal{W}$ is updated based on a dynamic adaption mechanism. This is triggered by the event that an EV is making charging reservations at the recommended CS within a time slot $\mathcal{K}$, then the traveling time $T_{\mathrm{ev}}^{\text {tra }}$ of the $\mathrm{EV}$ is compared with the value of estimation window $\mathcal{W}$ that is currently applied in the charging system. The larger value is updated as the new estimation window of $\mathcal{W}$.

The advantage is to gradually learn the charging demand distribution of EVs. This is to say, if most of EVs are with shorter $T_{\mathrm{ev}}^{\text {tra }}$ toward CSs recommended to them, a much urgent charging will be prepared. As such, the way to predict the CSs' charging availability will be with a tight $\mathcal{W}$ (or say smaller $\mathcal{W}$ ), such that the accuracy is adjusted with $\frac{\mathcal{W}}{\mathcal{H}}$.

\section{Prediction of Charging Availability Without EVs' Charging Reservations}

Here, as no EVs' charging reservations are available, the charging availability is computed solely based on the CSs' local queueing information. A set QLIST is defined to represent the available time of all charging slots locally at a CS.

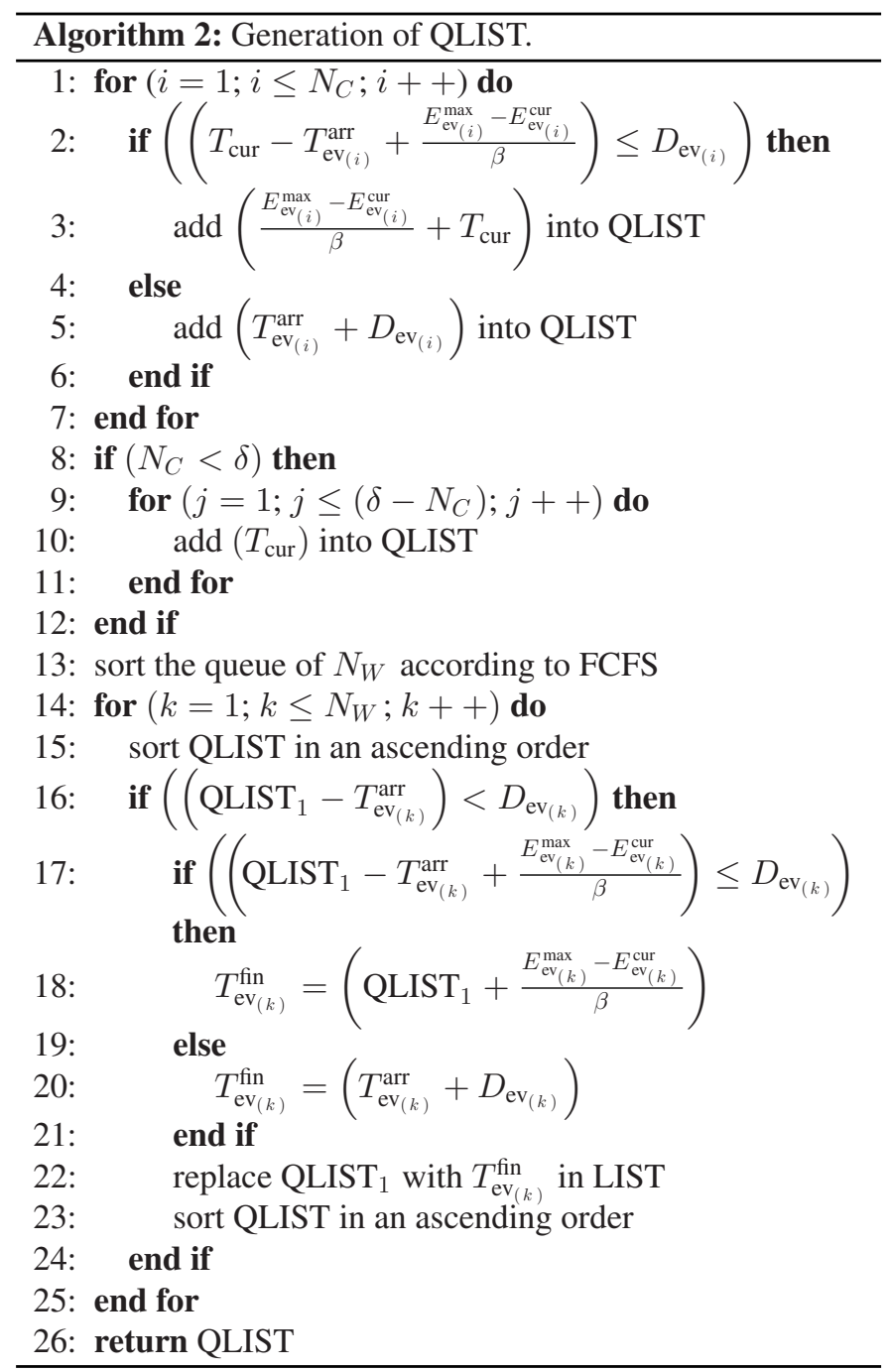

1) Generation of QLIST: As each CS has $\delta$ charging slots to charge parked EVs in parallel, we consider two types of queues localized at the CS. Here, EVs being charged are included in the queue of $N_{C}$, while those waiting for charging (due to all $\delta$ charging slots of a CS have been occupied by other EVs for charging) are characterized in the queue of $N_{W}$.

From line 1 at Algorithm 2, for each $\mathrm{EV}_{i}$ being charged, the time length $\left(\frac{E_{\mathrm{ev}_{(i)}}^{\max }-E_{\mathrm{ev}_{(i)}}^{\operatorname{cur}}}{\beta}\right)$ to fully recharge its battery (in the queue of $N_{C}$ ), will be compared with its parking duration $D_{\mathrm{ev}_{(i)}}$. The comparison outcome is applied to estimate the time that $\mathrm{EV}_{i}$ will take to finish its charging.

1) In one case, the condition $\left(\left(T_{\mathrm{cur}}-T_{\mathrm{ev}_{(i)}}^{\mathrm{arr}}+\right.\right.$ $\left.\left.\frac{E_{\mathrm{ev}_{(i)}}^{\max }-E_{\mathrm{ev}_{(i)}}^{\mathrm{cur}}}{\beta}\right) \leq D_{\mathrm{ev}_{(i)}}\right)$ implies that $\mathrm{EV}_{i}$ can be fully recharged before departure. Here, $\left(T_{\mathrm{cur}}-T_{\mathrm{ev}_{(i)}}^{\mathrm{arr}}\right)$ is the time duration to wait for charging since the arrival of $\mathrm{EV}_{i}$. As such, at line 3, the charging finish time (about when the charging of $\mathrm{EV}_{i}$ will finish) $T_{\mathrm{ev}_{(i)}}^{\mathrm{fin}}$ of $\mathrm{EV}_{i}$ is given by a summation of $\left(\frac{E_{\operatorname{ev}_{(i)}^{\max }}^{\operatorname{mat}}-E_{\mathrm{ev}(i)}^{\mathrm{cur}}}{\beta}+T_{\text {cur }}\right)$ only.

2) In another case, $T_{\operatorname{ev}_{(i)}}^{\mathrm{in}}$ is given by $\left(T_{\mathrm{ev}_{(i)}}^{\mathrm{arr}}+D_{\mathrm{ev}_{(i)}}\right)$ at line 5 , as the time slot that $\mathrm{EV}_{i}$ leaves from CS. 


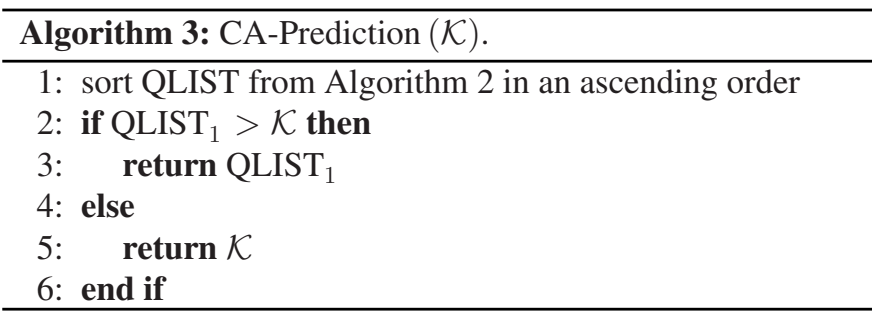

Furthermore, the presentation between lines 8 and 12 reflects a case that not all $\delta$ charging slots have been occupied by other EVs for charging. Therefore, it is easy to determine that there are still $\left(\delta-N_{C}\right)$ slots that can be reserved by incoming EVs for charging. As such, the available charging time for these unoccupied charging slots is all unified as $T_{\text {cur }}$.

Then, Algorithm 2 first sorts the queue of $N_{W}$ based on the FCFS order, by following the charging scheduling in Section III. Besides, QLIST that includes those EVs under charging will be sorted in an ascending order. Here, the earliest available time for charging at a CS is deemed as the first element in QLIST, and we denote that time as QLIST $_{1}$ (the first element of sorted QLIST).

In detail, to calculate the charging finish time $T_{\mathrm{ev}_{(k)}}^{\mathrm{in}}$ of each $\mathrm{EV}_{k}$ (in the queue of $N_{W}$ ), the earliest available time of charging slots is required to be known. In principle, it is crucial to consider $\mathrm{EV}_{k}$ that at least will be charged during its parking duration $D_{\mathrm{ev}_{(k)}}$ to involve calculation. This constraint is defined by $\left(\left(\mathrm{QLIST}_{1}-T_{\mathrm{ev}_{(k)}}^{\operatorname{arr}}\right)<D_{\mathrm{ev}_{(k)}}\right)$ at line 16.

1) Then from lines 17 and 21, either $\left(\right.$ QLIST $_{1}+$ $\left.\frac{E_{\mathrm{ev}_{(k)}}^{\max }-E_{\mathrm{ev}_{(k)}}^{\mathrm{cur}}}{\beta}\right)$ or $\left(T_{\mathrm{ev}_{(k)}}^{\operatorname{arr}}+D_{\mathrm{ev}_{(k)}}\right)$ calculates $T_{\mathrm{ev}_{(k)}}^{\mathrm{fin}}$, in particular, $\left(\mathrm{QLIST}_{1}-T_{\mathrm{ev}(k)}^{\mathrm{arr}}\right)$ is referred for $\mathrm{EV}_{k}$ to wait for charging.

2) Upon $T_{\mathrm{ev}_{(k)}}^{\mathrm{fin}}$ been given, $\mathrm{QLIST}_{1}$ will be replaced with $T_{\mathrm{ev}_{(k)}}^{\mathrm{fin}}$. Then, QLIST will be re-sorted in an ascending order upon processing each $\mathrm{EV}_{k}$ in the loop.

The aforementioned loop operation is finished when all $\mathrm{EV}_{k}$ (in the queue of $N_{W}$ ) have been processed and updated QLIST is generated.

2) Charging Availability Computing: Based on Algorithm 2 with QLIST being generated, the CS's local queueing information is computed to predict the charging availability associated with $\mathcal{K}$ in Algorithm 3. Here, as QLIST $_{1}$ is later than $\mathcal{K}$, the charging availability is represented as QLIST $_{1}$, and otherwise as $\mathcal{K}$. This depends on whether the CS will be available for charging at the time slot $\mathcal{K}$.

\section{E. Prediction of Charging Availability With EVs' Charging Reservations}

Recall that Algorithm 1 has already included a number of EVs into RLIST, which is an input for Algorithm 4. This guarantees that the charging availability of the CS is predicted by tracking the EVs that will reach the reserved CS within $\frac{\mathcal{W}}{\mathcal{H}}$ and the charging time of EVs that are parked there. Here, the latter information is provided by QLIST generated via Algorithm 2 and sorted in an ascending order.

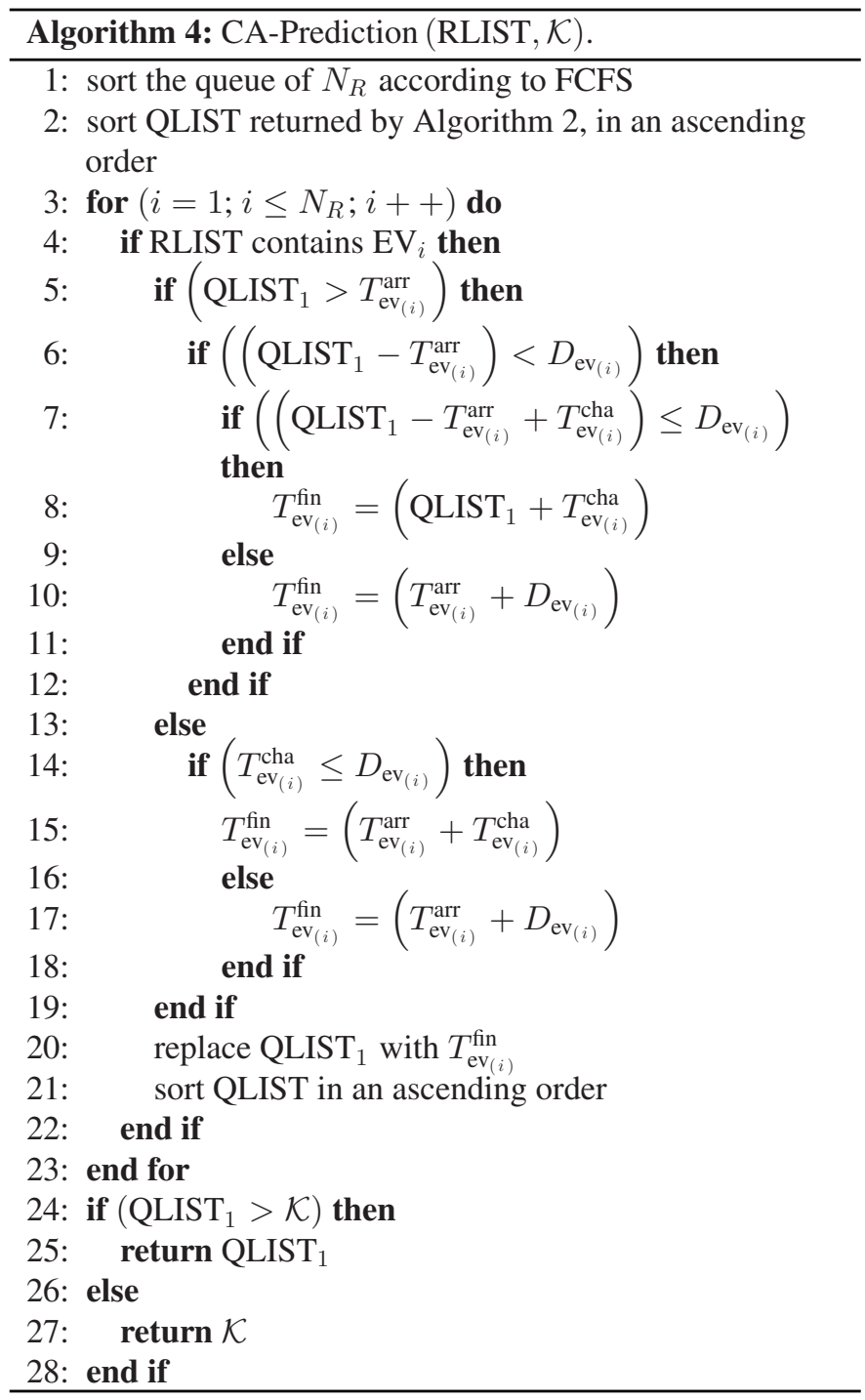

At line 5 in Algorithm 4, for each $\mathrm{EV}_{i}$ (in the queue of $N_{R}$ ) with its $T_{\mathrm{ev}_{(i)}}^{\mathrm{arr}}$ prior to the earliest available time for charging QLIST $_{1}, \mathrm{EV}_{i}$ will be taken into account for the update of QLIST. This means that only those EVs (in the queue of $N_{R}$ ) arriving later than QLIST $_{1}$ will not have an influence on QLIST. Note that QLIST has been previously sorted in an ascending order. This guarantees that the earliest time that one of the charging slots will be free, it is ready for taking the subsequent EV's charging.

1) In one case, the condition ( $\left.\mathrm{QLIST}_{1}>T_{\mathrm{ev}_{(i)}}^{\mathrm{arr}}\right)$ at line $5 \mathrm{im}$ plies that $T_{\mathrm{ev}_{(i)}}^{\operatorname{arr}}$ is prior to the earliest available time $\operatorname{LIST}_{1}$. This causes the charging finish time $T_{\mathrm{ev}_{(i)}}^{\mathrm{fin}}$ to be calculated by summating QLIST $_{1}$ and the expected charging time $T_{\mathrm{ev}_{(i)}}^{\mathrm{cha}}$.

In particular, at line 7, the condition (( $\mathrm{QLIST}_{1}-T_{\mathrm{ev}_{(i)}}^{\mathrm{arr}}+$ $\left.\left.T_{\mathrm{ev}_{(i)}}^{\text {cha }}\right) \leq D_{\mathrm{ev}_{(i)}}\right)$ implies that within the parking duration $D_{\mathrm{ev}_{(i)}}, \mathrm{EV}_{i}$ could be fully recharged. Recall that $\left(\mathrm{QLIST}_{1}-T_{\mathrm{ev}_{(i)}}^{\mathrm{arr}}\right)$ is the time to wait until the charging is started. In this context, given by the cases at lines 7 and 9, $T_{\mathrm{ev}_{(i)}}^{\mathrm{fin}}$ is given by $\left(\mathrm{QLIST}_{1}+T_{\mathrm{ev}_{(i)}}^{\mathrm{cha}}\right)$ or $\left(T_{\mathrm{ev}_{(i)}}^{\operatorname{arr}}+D_{\mathrm{ev}_{(i)}}\right)$. 


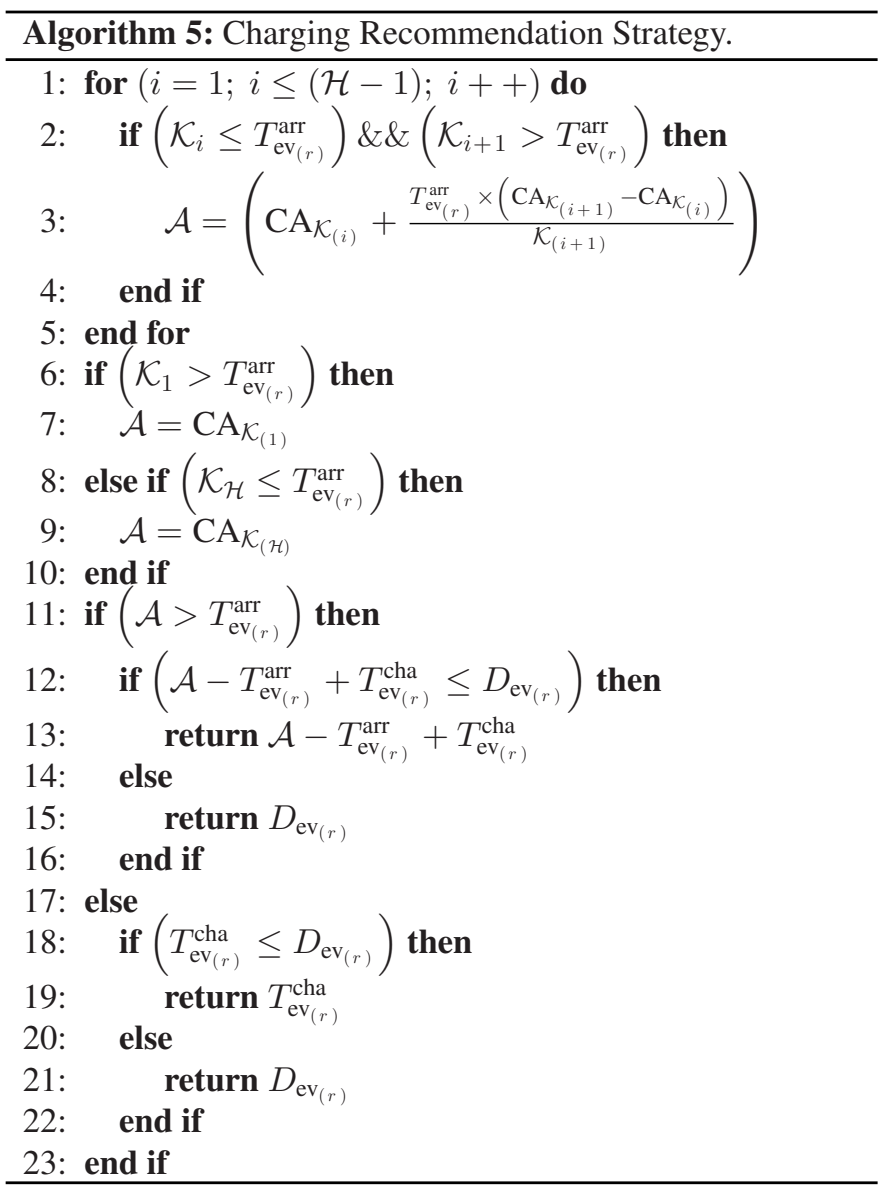

Note that as the condition given by $\left(\left(\mathrm{QLIST}_{1}-T_{\mathrm{ev}_{(i)}}^{\mathrm{arr}}\right)<\right.$ $\left.D_{\mathrm{ev}_{(i)}}\right)$ at line 6 , we only consider that $\mathrm{EV}_{i}$ could be charged before $D_{\mathrm{ev}_{(i)}}$ to involve the calculation.

2) In another case, $T_{\mathrm{ev}_{(i)}}^{\mathrm{fin}}$ is calculated by considering $T_{\mathrm{ev}_{(i)}}^{\mathrm{arr}}$, $T_{\mathrm{ev}_{(i)}}^{\mathrm{cha}}$, and $D_{\mathrm{ev}_{(i)}}$ following the calculations at lines 15 and 17. This only happens when $\left(\mathrm{QLIST}_{1} \leq T_{\mathrm{ev}_{(i)}}^{\mathrm{arr}}\right)$, meaning that the CS has already been available for charging when $\mathrm{EV}_{i}$ arrives.

By replacing QLIST ${ }_{1}$ with each $T_{\mathrm{ev}_{(i)}}^{\mathrm{fin}}$ in each loop round, QLIST will be dynamically updated. Furthermore, QLIST will be sorted in an ascending order after processing each $\mathrm{EV}_{i}$, such that the first element QLIST $_{1}$ is updated. The loop operation ends when all $\mathrm{EV}_{i}$ (in the queue of $N_{R}$ ) have been processed.

\section{F. Charging Recommendation}

Here, $\mathrm{EV}_{r}$ is denoted as the EV that needs to make a charging recommendation, other than those EVs that are either being parked or on the move. Two bounding time slots can be obtained via the condition at line 2 of Algorithm 5, such that the arrival time of $\mathrm{EV}_{r}$, denoted as $T_{\mathrm{ev}_{(r)}}^{\mathrm{arr}}$, is between these two time slots $\mathcal{K}_{i}$ and $\mathcal{K}_{i+1}$. In this case, the outcome of the charging availability is then passed to a temporary variable $\mathcal{A}$, with $\mathcal{A}=\left(\mathrm{CA}_{\mathcal{K}_{i}}+\frac{T_{\mathrm{ev}(r)}^{\mathrm{arr}} \times\left(\mathrm{CA}_{\mathcal{K}_{i+1}}-\mathrm{CA}_{\mathcal{K}_{i}}\right)}{\mathcal{K}_{i+1}}\right)$ at line 3, considering a ratio between $T_{\mathrm{ev}_{(r)}}^{\mathrm{arr}}$ and $\mathcal{K}_{i+1}$. From this calculation, it is aimed to capture the charging availability upon its arrival time $\mathrm{EV}_{(r)}$ that is between $\mathcal{K}_{i}$ and $\mathcal{K}_{i+1}$.

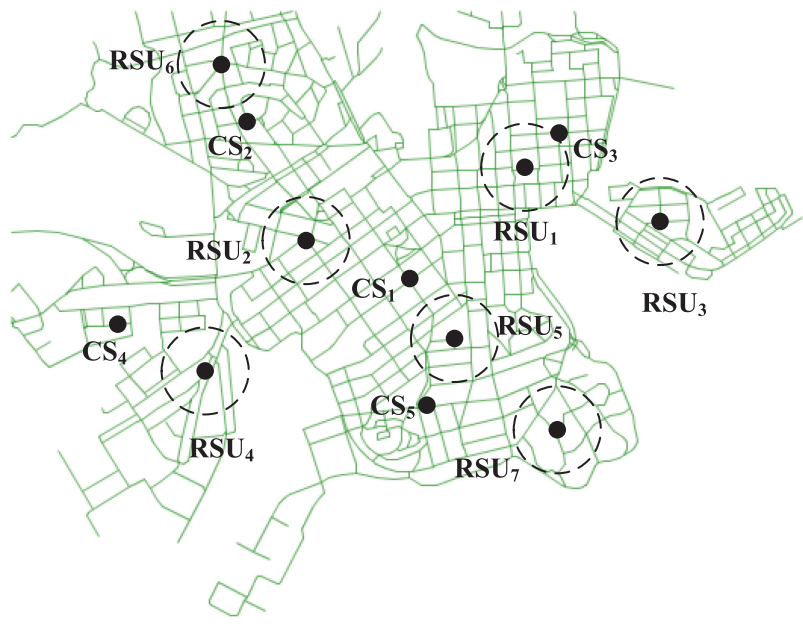

Fig. 5. Helsinki city scenario.

There are also two cases if $T_{\mathbf{e v}_{(r)}}^{\operatorname{arr}}$ is out of the bound of the estimation window $\mathcal{W}$.

1) Due to that $T_{\mathrm{ev}_{(r)}}^{\mathrm{arr}}$ is earlier than the earliest estimation time slot in entries $\mathcal{H}$, denoted as $\mathcal{K}_{1}$, the charging availability upon the arrival of $\mathrm{EV}_{r}$ is given by $\mathrm{CA}_{\mathcal{K}_{(1)}}$ at line 7.

2) Besides, due to that $T_{\mathrm{ev}_{(r)}}^{\mathrm{arrr}}$ is later than the latest time slot in entries $\mathcal{H}$, the charging availability in this case is given by $\mathrm{CA}_{\mathcal{K}_{(\mathcal{H})}}$ at line 9 .

Next, $\mathrm{EV}_{r}$ will predict an expected time for which it would stay at the recommended CS before the parking deadline by considering its parking duration $D_{\mathrm{ev}_{(r)}}$.

1) Basically, if $\mathrm{EV}_{r}$ arrives later than $\mathcal{A}$, this means it still needs to wait for additional time until a charging slot is available. In this case, the condition $\left(\mathcal{A}-T_{\mathrm{ev}_{(r)}}^{\mathrm{arr}}+\right.$ $\left.T_{\mathrm{ev}_{(r)}}^{\mathrm{cha}} \leq D_{\mathrm{ev}_{(r)}}\right)$ indicates $\mathrm{EV}_{r}$ can be fully recharged within the parking deadline $D_{\mathrm{ev}_{(r)}}$; thus, its expected staying time is calculated by $\left(\mathcal{A}-T_{e v}^{\text {arr }}+T_{e v_{(r)}}^{\text {cha }}\right)$ at line 13 . Otherwise, only $D_{\mathrm{ev}_{(r)}}$ is referred as the staying time at line 14

2) Such a policy between lines 18 and 22 can be also applied to the case if $\mathrm{EV}_{r}$ arrives no later than $\mathcal{A}$. In this case, as $\mathrm{EV}_{r}$ does not need to wait for additional time to start charging, the comparison is just between $T_{\mathrm{ev}_{(r)}}^{\mathrm{arr}}$ and $D_{\mathrm{ev}_{(r)}}$.

\section{Performance Evaluation}

\section{A. Scenario Configuration}

The entire system for EV charging is built in Opportunistic Network Environment [21]. In Fig. 5, the default scenario with $4500 \times 3400 \mathrm{~m}^{2}$ area is shown as the downtown area of Helsinki city in Finland. $N_{\mathrm{ev}}=300$ EVs with $S_{\mathrm{ev}}=[30 \sim 50]$ $\mathrm{km} / \mathrm{h}$ variable moving speed are initialized considering road safety in a city. The configuration of EVs follows the charging specification of Hyundai BlueOn, with a maximum electricity capacity of $16.4 \mathrm{kWh}$, max traveling distance $140 \mathrm{~km}$, and SOC [15 45]\%. Besides, $N_{\mathrm{cs}}=5 \mathrm{CSs}$ are provided with sufficient electric energy and $\delta=5$ charging slots through entire simulation, using the fast charging rate of $\beta=62 \mathrm{~kW} . R=300 \mathrm{~m}$ radio coverage is applied for $N_{\mathrm{mec}}=7 \mathrm{RSUs}$ and $N_{\mathrm{ev}}=300 \mathrm{EVs}$. 


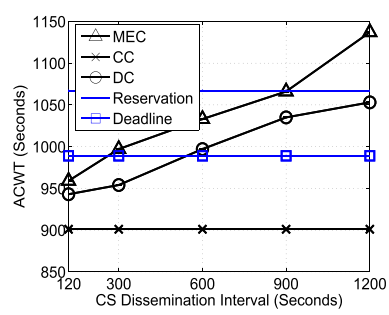

(a)

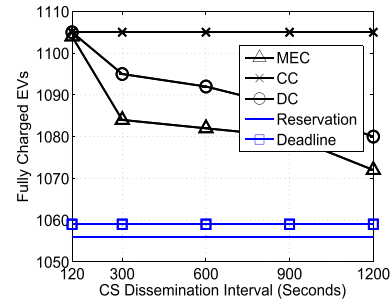

(b)

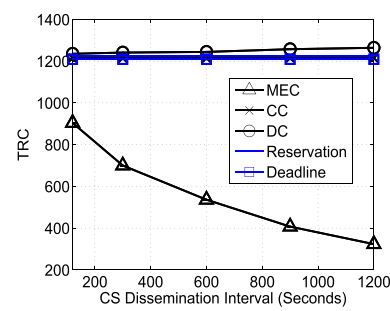

(c)

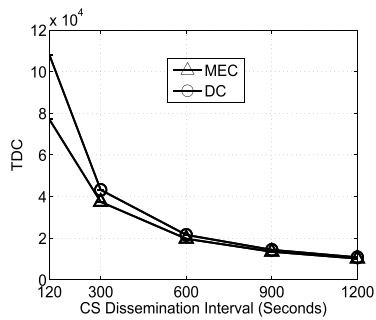

(d)

Fig. 6. Influence of CS dissemination interval $\Delta$. (a) ACWT. (b) Fully charged EVs. (c) TRC. (d) TDC.

The default dissemination interval of CS's charging availability is $\Delta=120 \mathrm{~s}$, and the simulation time is $43200 \mathrm{~s}=12 \mathrm{~h}$.

The following schemes are evaluated for comparison.

1) $M E C$ : The proposed charging recommendation scheme in Section IV, based on the MEC framework in Section III.

2) $C C$ and $D C$ : They are with the same charging recommendation scheme with MEC, but with centralized and distributed cloud computing framework.

3) Reservation [10]: Previous works take the EVs' charging reservation to predict the CSs' charging availability, however, not addressing the EVs' parking deadline. Here, the cloud computing framework is positioned.

4) Deadline [11]: Previous works taking the parking deadline into the account of charging recommendation, based on the cloud computing framework. This scheme differs from the $\mathrm{CC}$ for the computation intelligence to predict CSs' charging availability.

The simulation evaluates metrics at the EV and CS sides as well as communication costs at the system level.

1) Average Charging Waiting Time (ACWT): The average period between the time an $\mathrm{EV}$ arrives at the recommended $\mathrm{CS}$ and the time it finishes (full) recharging its battery. This is the performance metric at the EV side.

2) Fully Charged EVs: The total number of fully charged EVs; this is the performance metric at the CS side.

3) Total Reservation Cost (TRC): The total number signaling reported for EV's charging reservations to the GC. In MEC, this counts for the signaling from RSUs to the GC, whereas other schemes count from EVs to the GC.

4) Total Dissemination Cost (TDC): In MEC, this counts for the signaling from RSUs to the EVs, whereas in DC, this counts from GC to EVs.

\section{B. Performance Results}

1) Influence of CS Dissemination Interval $\Delta$ : Results in Fig. 6(a) and (b) show that a frequent dissemination interval helps to maintain the optimality of the charging recommendation. This means that as the information is replaced at RSUs frequently, EVs that have passed by would fetch the cached information that is more fresh. In comparison to DC, the CC achieves the better performance by making decision using a seamless cellular network communication, compared to the opportunistic communication between RSUs and EVs as applied in the MEC system. Furthermore, concerning the feature of charging recommendation, the $\mathrm{CC}$ outperforms reservation and deadline, thanks to decoupling the decision making within a small-time interval $\mathcal{W}$. It is also observed that the deadline outperforms reservation, as the former takes the EVs' parking deadline into account.

In Fig. 6(c), the MEC-based system is with decreased TRC, which follows the analysis in Section III. However, other compared charging recommendations with the cloud-based system are with a much higher TRC. The benefit of reduced TRC is from the aggregation and mining functions at RSUs, which filter invalid EVs' charging reservations (which to be not uploaded to the GC) for computation. Besides, the dissemination cost is shown in Fig. 6(d), where the cost in MEC is lower than in DC-based systems (with $\Delta=120 \mathrm{~s}$ ). This shows the efficiency of using on-demand and short-range wireless communication in the MEC-based system together with access control, compared to the long-range cellular link and broadcasting communication in the DC-based system. In the following sections, DC is excluded, while only the nature of charging recommendation solutions is discussed.

2) Influence of Parking Deadline $D_{\text {ev }}$ : In Fig. 7(b), a longer parking deadline $D_{\mathrm{ev}}$ increases the fully charged EVs. This is generally referred to the situation that EVs being parked at CSs will have much chance to be fully charged, compared to the case with $1200 \mathrm{~s}$ parking deadline, while such increase brings increased ACWT in Fig. 7(a) as well. In Fig. 7(c), it is observed that a shorter parking deadline leads to a much higher TRC. This is because of those EVs that are not fully charged and would subsequently need charging after a shorter period. As such, the charging reservation is increased corresponding to such frequent charging demands.

Apart from the above-mentioned general observation, further details are comparable in the cases of 5 and 7 charging slots. The latter case alleviates the charging congestion at CSs; as such, it delivers a lower AWCT and higher fully charged EVs as well as reduced TRC (more significant in the case of 1200 s parking deadline).

3) Influence of EV Density $N_{\mathrm{ev}}$ : In Fig. 8(a), the AWCT is increased from the case of $100 \mathrm{EVs}$, as more EVs will be fully charged (with 300 and 500 EVs). However, Fig. 8(b) shows that the fully charged EVs are first increased from 100 to $300 \mathrm{EVs}$ cases, and then decreases from 300 to $500 \mathrm{EVs}$ cases. This reflects the $500 \mathrm{EVs}$ case results in severe charging congestion, so some EVs are not fully charged. Such an outcome is also associated with the TRC, wherein Fig. 8(c) shows the TRC in 


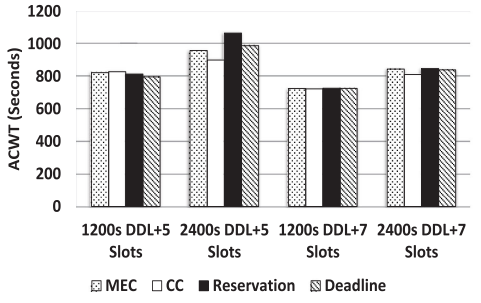

(a)

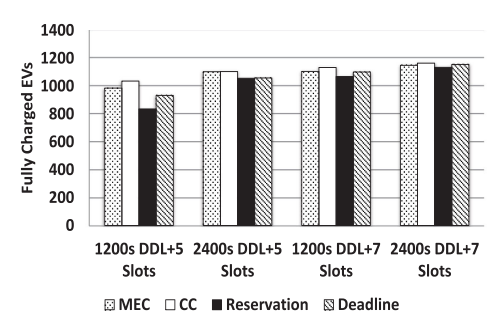

(b)

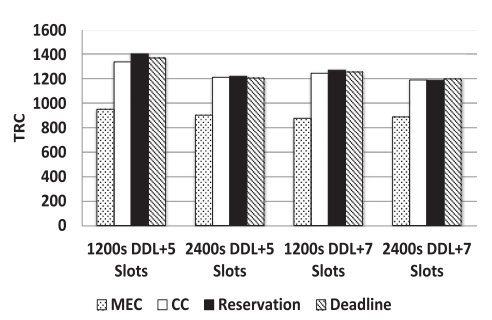

(c)

Fig. 7. Influence of EVs' parking deadline $D_{\text {ev }}$. (a) ACWT. (b) Fully charged EVs. (c) TRC.

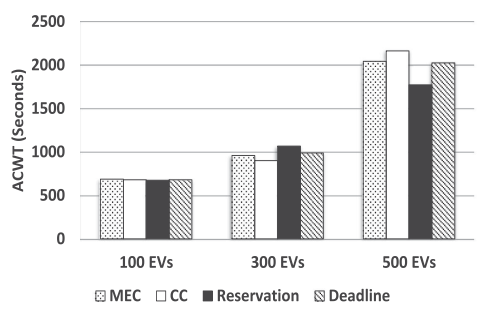

(a)

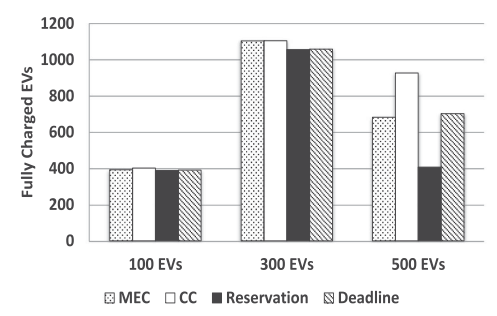

(b)

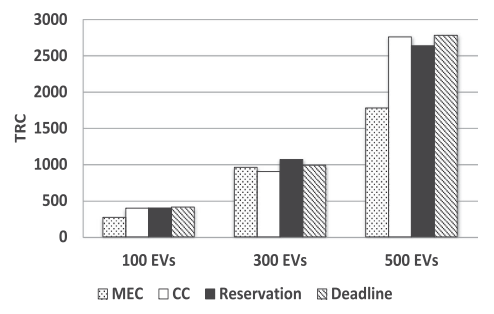

(c)

Fig. 8. Influence of EVs' density $N_{\mathrm{ev}}$, with $\delta=5$ charging slots. (a) ACWT. (b) Fully charged EVs. (c) TRC.

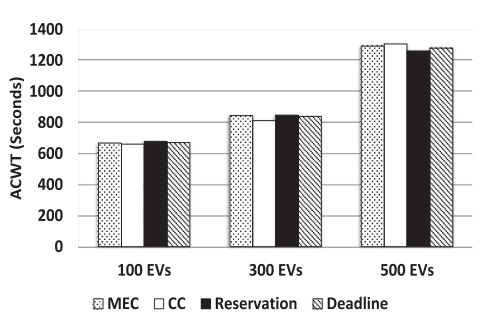

(a)

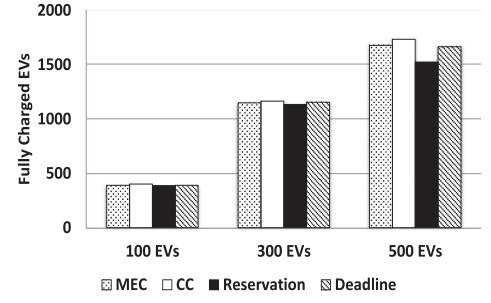

(b)

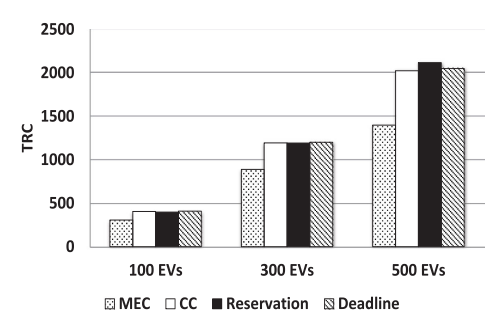

(c)

Fig. 9. Influence of EVs' density $N_{\text {ev }}$, with $\delta=7$ charging slots. (a) ACWT. (b) Fully charged EVs. (c) TRC.

the case of $500 \mathrm{EVs}$, is much higher than the fully charged EVs in the same case of Fig. 8(b). The mismatch is because of the EVs that were not fully charged but later need charging (with additional charging reservations sent).

If setting 7 charging slots at each CS, where the fully charged EVs is increased in Fig. 9(b), along with increased ACWT in Fig. 9(a). Compared with that in Fig. 8(b) where there is a decrease of fully charged EVs from 300 to $500 \mathrm{EVs}$ cases, the situation here implies the effect of parking deadline with limited charging infrastructures. Of course, the MEC-based system still achieves the lowest TRC in Fig. 9(c), similar to the previous observation.

\section{CONCLUSION}

This paper investigated EV charging recommendation via MEC architecture, with RSUs positioned physically and MEC functions virtually to help with information dissemination and collection. The information access control, aggregation, and mining are enabled at MEC servers, while the charging recommendation takes the EV's charging reservation and its parking deadline into account. Results show that the proposed solution achieves a comparable performance in terms of charging waiting time as a benefit to the user, and a number of fully charged EVs as a benefit to the service provider. Future works would be on integration of the power network.

With the ever increasing penetrations in EVs, the resultant charging energy imposed on the electricity network could lead to grid issues, such as voltage limits violation, transformer overloading, and feeder overloading at various voltage levels. Coordination of the charging energy with a renewable energy source provides a more straightforward approach to cope with the potential network issues as mentioned previously. Future works would be on the integration of power network to achieve an interdisciplinary work on ICT, route planning, and energy integration.

\section{REFERENCES}

[1] Y. Cao, N. Wang, G. Kamel, and Y.-J. Kim, "An electric vehicle charging management scheme based on publish/subscribe communication framework," IEEE Syst. J., vol. 11, no. 3, pp. 1822-1833, Sep. 2017.

[2] J. C. Mukherjee and A. Gupta, "A review of charge scheduling of electric vehicles in smart grid," IEEE Syst. J., vol. 9, no. 4, pp. 1541-1553, Dec. 2015.

[3] E. Rigas, S. Ramchurn, and N. Bassiliades, "Managing electric vehicles in the smart grid using artificial intelligence: A survey," IEEE Trans. Intell. Transp. Syst., vol. 16, no. 4, pp. 1619-1635, Aug. 2015.

[4] S.-N. Yang, W.-S. Cheng, Y.-C. Hsu, C.-H. Gan, and Y.-B. Lin, "Charge scheduling of electric vehicles in highways," Elsevier Math. Comput. Model., vol. 57, no. 1112, pp. 2873-2882, Jun. 2013. 
[5] M. Gharbaoui, L. Valcarenghi, R. Bruno, B. Martini, M. Conti, and P. Castoldi, "An advanced smart management system for electric vehicle recharge," in Proc. IEEE Int. Elect. Veh. Conf., Greenville, SC, USA, Mar. 2012.

[6] F. Hausler, E. Crisostomi, A. Schlote, I. Radusch, and R. Shorten, "Stochastic park-and-charge balancing for fully electric and plug-in hybrid vehicles," IEEE Trans. Intell. Transp. Syst., vol. 15, no. 2, pp. 895-901, Apr. 2014.

[7] Y. Cao, N. Wang, and G. Kamel, "A publish/subscribe communication framework for managing electric vehicle charging," in Proc. IEEE Int. Conf. Connected Veh. Expo., Vienna, Austria, Nov. 2014, pp. 318-324.

[8] Q. Tang, K. Wang, Y.-S. Luo, and K. Yang, "Congestion balanced green charging networks for electric vehicles in smart grid," in Proc. IEEE Int. Conf. Commun., Paris, France, 2017.

[9] H. Qin and W. Zhang, "Charging scheduling with minimal waiting in a network of electric vehicles and charging stations," in Proc. 8th ACM Int. Workshop Veh. Internetworking, Las Vegas, NV, USA, Sep. 2011, pp. 51-60.

[10] Y. Cao et al., "Towards efficient, scalable and coordinated on-the-move EV charging management," IEEE Wireless Commun., vol. 24, no. 2, pp. 66-73, Apr. 2017.

[11] Y. Cao, T. Wang, O. Kaiwartya, G. Min, N. Ahmad, and A. H. Abdullah, "An EV charging management system concerning drivers' trip duration and mobility uncertainty," IEEE Trans. Syst., Man, Cybern., Syst., vol. 48, no. 4, pp. 596-607, Apr. 2016.

[12] G. Jia, G. Han, J. Jiang, N. Sun, and K. Wang, "Dynamic resource partitioning for heterogeneous multi-core-based cloud computing in smart cities," IEEE Access, vol. 4, pp. 108-118, 2016.

[13] M. T. Beck, M. Werner, S. Feld, and S. Schimper, "Mobile edge computing: A taxonomy," in Proc. 6th Int. Conf. Adv. Future Internet, Citeseer, 2014.

[14] M. Rashidi, I. Batros, T. Madsen, M. Riaz, and T. Paulin, "Placement of road side units for floating car data collection in highway scenario," in Proc. IEEE Int. Congr. Ultra Modern Telecommun. Control Syst., St. Petersburg, Russia, Oct. 2012.

[15] K. Ghafoor, J. Lloret, K. Bakar, A. Sadiq, and S. Mussa, "Beaconing approaches in vehicular ad hoc networks: A survey," Wireless Pers. Commun., vol. 73, no. 3, pp. 885-912, 2013.

[16] A. D'Elia et al., "Impact of interdisciplinary research on planning, running, and managing electromobility as a smart grid extension," IEEE Access, vol. 3, pp. 2281-2305, 2015.

[17] L. Bedogni et al., "An integrated simulation framework to model electric vehicle operations and services," IEEE Trans. Veh. Technol., vol. 65, no. 8 , pp. 5900-5917, Aug. 2016.

[18] Y. Cao, C. Long, T. Jiang, and S. Mao, "Share communication and computation resources on mobile devices: A social awareness perspective," IEEE Wireless Commun., vol. 23, no. 4, pp. 52-59, Aug. 2016.

[19] Y. Cao and Z. Sun, "Routing in delay/disruption tolerant networks: A taxonomy, survey and challenges," IEEE Commun. Surveys Tut., vol. 15, no. 2, pp. 654-677, Apr--Jun. 2013.

[20] P. T. Eugster, P. A. Felber, R. Guerraoui, and A.-M. Kermarrec, "The many faces of publish/subscribe," ACM Comput. Surveys,vol. 35, no. 2, pp. 114-131, Jun. 2003.

[21] A. Keränen, J. Ott, and T. Kärkkäinen, "The ONE simulator for DTN protocol evaluation," in Proc. 2nd Int. Conf. Simul. Tools Techn., Rome, Italy, Mar. 2009

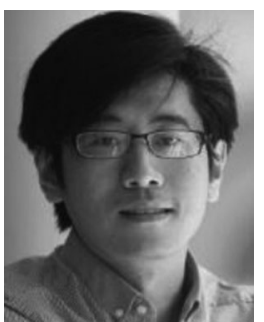

Yue Cao (M'16) received the Ph.D. degree from the Institute for Communication Systems (ICS), 5G Innovation Centre, University of Surrey, Guildford, U.K., in 2013.

He was a Research Fellow with the ICS until September 2016, and a Lecturer with the Department of Computer and Information Sciences, Northumbria University, Newcastle upon Tyne, U.K., until July 2017, and has been the Senior Lecturer since $\mathrm{Au}$ gust 2017. His research interest focuses on intelligent mobility.

Dr. Cao is the Associate Editor for the IEEE ACCESS and the International Journal of Vehicular Telematics and Infotainment Systems.

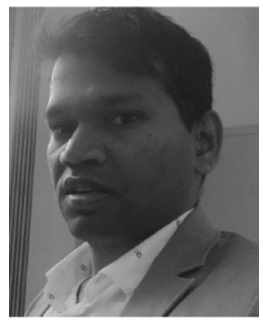

Omprakash Kaiwartya (M'14) received the Ph.D. degree in computer science from the School of Computer and Systems Sciences, Jawaharlal Nehru University, New Delhi, India, in 2015.

Since May 2018, he has been a Lecturer with the School of Science and Technology, Nottingham Trent University, Nottingham, U.K. In 2017, he was a Research Associate with the Department of Computer and Information Sciences, Northumbria University, U.K., and in 2016, he was a Postdoctoral Research Fellow with the Faculty of Computing, Universiti Teknologi Malaysia, Johor Bahru, Malaysia. His research interests includes Internet of connected vehicles, electronic vehicles charging management, and IoT use cases in sensor networks.

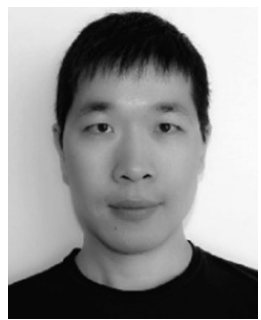

Yuan Zhuang (M'16) received the bachelor's degree in information engineering and the master's degree in microelectronics and solid-state electronics from Southeast University, Nanjing, China, in 2008 and 2011, respectively, and the Ph.D. degree in geomatics engineering from the University of Calgary, Calgary, AB, Canada, in 2015 .

$\mathrm{He}$ is currently a Lead Scientist with Bluvision, Inc. (a part of HID Global). His current research interests include IoT-based asset tracking and condition monitoring, real-time location system, pedestrian navigation, and multisensors integration.

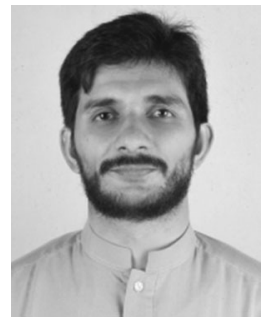

Naveed Ahmad received the B.Sc. (Hons.) degree in computer sciences from the University of Peshawar, Peshawar, Pakistan, in 2007, and the Ph.D. degree in electronics and electrical engineering from the Institute of Communication System, University of Surrey, Guildford, U.K., in 2013.

$\mathrm{He}$ is currently an Assistant Professor with the Department of Computer Science, University of Peshawar. His research interests include routing, security, and privacy in emerging networks, such as vehicular ad hoc networks, delay-tolerant networks,

and Internet of Things

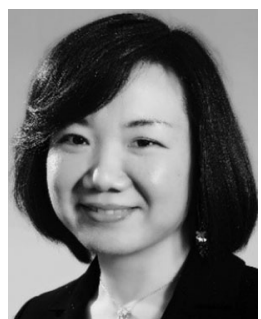

Yan Sun received the B.Eng. degree in telecommunications engineering from the Beijing University of Posts and Telecommunications, Beijing, China, in 2001, and the M.Sc. and Ph.D. degrees in electronic engineering from the Queen Mary University of London, London, U.K., in 2003 and 2009, respectively.

In 2001, she joined Siemens, Ltd., China, as a Network Optimization Engineer, and rejoined in 2003, as a System Engineer in R\&D after receiving the master's degree in the U.K. She was with Siemens (later Nokia Siemens Networks, Ltd.) as a Product Manager. In 2009, she joined the Queen Mary University of London as a Lecturer. Her current research interests include ad hoc networks, software-defined networks, and mobile healthcare networks.

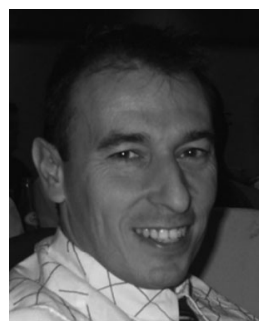

Jaime Lloret (M'07-SM'10) received the M.Sc. degree in physics in 1997, the postgraduate master's in corporative networks and systems integration from the Department of Communications in 1999, and the M.Sc. degree in electronic engineering in 2003, all from the University of Valencia, Valencia, Spain, and the Ph.D. degree in telecommunication engineering (Dr. Ing.) from the Polytechnic University of Valencia, Valencia, Spain, in 2006

He is currently an Associate Professor with the Universitat Politecnica de Valencia, Valencia, Spain. Dr. Lloret was the Chair of the Internet Technical Committee (IEEE Communications Society and Internet Society) and Working Group of the Standard IEEE 1907.1. He is an Editor-in-Chief for the Ad Hoc and Sensor Wireless Networks and the Associate Editor of 46 international journals (16 ISI). 